\title{
Selection bias: maintaining less-differentiated T cells for adoptive immunotherapy
}

\author{
Yang Xu and Gianpietro Dotti \\ Department of Microbiology and Immunology, Lineberger Cancer Center, University of North Carolina, Chapel Hill, North Carolina, USA.
}

\begin{abstract}
The clinical application of T cell immunotherapy depends on ex vivo modification and expansion of T cells for adoptive transfer. In preclinical models, the use of a purified, naive $T$ cell subset enhances persistence and antitumor immunity; however, the majority of clinical studies rely on modification of mixed populations of $T$ cells that contain only a small subset of highly functional T cells with less-differentiated phenotype. In this month's issue of the $J C I$, Klebanoff and colleagues uncover a Fas-mediated interaction between naive $T$ cells and antigen-experienced $T$ cells that drives differentiation and impairs adoptive immunotherapy. Further, they show that blockade of Fas signaling enhances antitumor immunity and increases survival in a mouse model of melanoma. Their work supports a growing body of evidence that the use of naive T cells enhances the efficacy of adoptive T cell therapy and suggests a new therapeutic strategy for preserving less-differentiated $\mathrm{T}$ cell populations.
\end{abstract}

\section{Generating T cells} for immunotherapy

Adoptive transfer of $\mathrm{T}$ lymphocytes engineered to achieve tumor specificity through the genetic insertion of either $\mathrm{T}$ cell receptors or chimeric antigen receptors (CARs) allows remarkable control of disseminated tumors across multiple phase I/II clinical studies in the academic setting $(1,2)$. Some of these approaches are now further developed in multicenter studies supported by pharmaceutical companies. Manufacturing of engineered $\mathrm{T}$ cells is a key element for the realistic execution of multicenter clinical studies and for the future use of these cells in the clinical practice. Manufacturing of $\mathrm{T}$ cells is generally centralized to ensure reproducibility and aims at implementing standard operating procedures with minimized complexity. To this end, peripheral blood mononuclear cells (PBMCs) are frequently preferred for generating engineered $\mathrm{T}$ cell products. PBMCs are indeed readily obtained by gradient centrifugation and contain all cir- culating $\mathrm{T}$ cell subsets, from naive $\mathrm{T}$ cells to memory T cells, susceptible to effective transduction by viral vectors encoding the transgene following activation with CD3/ CD28 crosslinking antibodies and cytokines. Starting from PBMCs, T cell products can be obtained within two weeks of ex vivo culture, a time schedule compatible with an effective clinical application in many patients with refractory and resistant malignancies.

\section{To select or not to select - that is the question}

In the current issue of the JCI, Klebanoff et al. highlight that the complexity of the immune system may not be easily recapitulated using simplified approaches and that a more sophisticated manipulation of $\mathrm{T}$ cell subsets within the infused $\mathrm{T}$ cell products may affect clinical outcome (3). This last observation stems from a long history of mouse model studies showing how less-differentiated $\mathrm{T}$ cell subsets from naive $T$ cells $\left(\mathrm{T}_{\mathrm{N}}\right.$ cells) to central memory $\mathrm{T}$

Related Article: p. 318

Conflict of interest: The authors have declared that no conflict of interest exists

Reference information: J Clin Invest. 2016;126(1):35-37. doi:10.1172/JCI85631.

cells $\left(\mathrm{T}_{\mathrm{CM}}\right.$ cells) display superior antitumor capacity and persistence compared with more-differentiated $\mathrm{T}$ cells, such as effector-memory $\mathrm{T}$ cells ( $\mathrm{T}_{\mathrm{EM}}$ cells) or effector $\mathrm{T}$ cells ( $\mathrm{T}_{\mathrm{E}}$ cells) (4). More recently, another subset of memory $\mathrm{T}$ cells, identified as $\mathrm{T}$ stem cell memory cells ( $\mathrm{T}_{\mathrm{SCM}}$ cells), claims properties superior even to $\mathrm{T}_{\mathrm{N}}$ and $\mathrm{T}_{\mathrm{CM}}$ cells (5). Clinical observations corroborate the relevance of preserving primitive $\mathrm{T}$ cell subsets within infused $\mathrm{T}$ cell products to ensure $\mathrm{T}$ cell expansion and potentially long-term engraftment $(6,7)$.

The composition of $\mathrm{T}$ cell subsets found in PBMCs of cancer patients is largely affected by several factors, including age, disease, and type of chemotherapy treatments received before blood procurement. All these factors contribute to creating very heterogeneous $\mathrm{T}$ cell products when whole PBMCs are used as a starting material. One could argue that the high response rate in both adult and pediatric patients with acute lymphoblastic leukemia infused with CD19-specific CAR-T cells supports the use of PBMCs as a starting source of $\mathrm{T}$ cells, regardless of their heterogeneity, as they contain all the $\mathrm{T}$ cell subsets needed for an effective immune response $(2,8,9)$. However, a more precise composition of the $\mathrm{T}$ cell products and in particular the enrichment in T cells with the highest potential for engraftment may become particularly relevant in other clinical settings, specifically in most solid tumors, in which the response rate to adoptive $\mathrm{T}$ cell therapies is currently significantly inferior compared with that for lymphoblastic leukemia.

\section{Maintaining undifferentiated T cell populations}

How can we preserve more immature and bona fide highly functional $\mathrm{T}$ cell subsets within infusion products without applying complex standard operating procedures? Activation, genetic manipulation, and ex vivo expansion of $\mathrm{T}$ cells inevitably induce their differentiation. This differentiation 

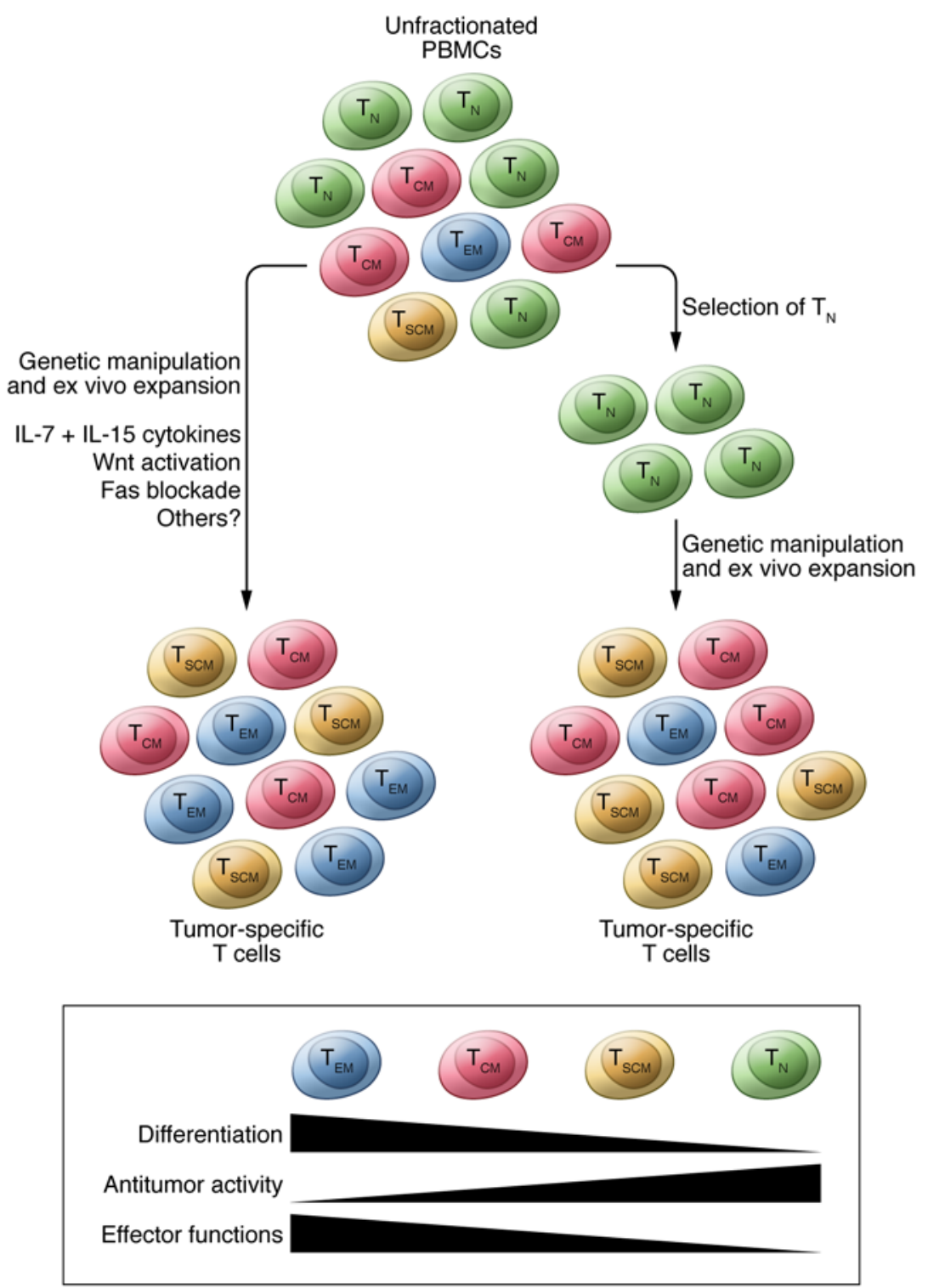

process can be stalled through manipulations during the expansion phase using cytokines, such as IL-7 and IL-15 instead of IL-2, to preserve more $\mathrm{T}_{\mathrm{SCM}}$ cells $(7,10)$. In addition, activation of the Wnt-signaling pathway has been implied to delay $\mathrm{T}$ cell differentiation $(11,12)$. However, in the current issue of the JCI, Klebanoff et al. further elucidate the complexity of $\mathrm{T}$ cell interactions in vitro showing in both mouse and human that memory $\mathrm{T}$ cells $\left(\mathrm{T}_{\mathrm{MEM}}\right.$ cells) actively induce "precocious differentiation" of $\mathrm{T}_{\mathrm{N}}$ cells when they are activated and expanded together. In the presence of $\mathrm{T}_{\mathrm{MEM}}$ cells, $\mathrm{T}_{\mathrm{N}}$ cell progenies acquire enhanced effector functions, such as expression of granzyme B and the ability to produce IFN- $\gamma$ upon restimulation. $\mathrm{T}_{\mathrm{MEM}}$ cells also mediate global transcriptional
Figure 1. Schematic representation of the generation of engineered $T$ cells for adoptive immunotherapy. Unfractionated PBMCs containing $T_{N}, T_{S C M}, T_{C M}$, and $T_{E M}$ cells are frequently used as starting material to generate genemanipulated T cell products for adoptive immunotherapy in cancer patients. To preserve more immature T cell subsets, the manipulation of culture conditions by using different cocktails of cytokines or by activating or inhibiting specific pathways has been developed. In the current issue of the $J C I$, Klebanoff et al. suggest that the selection of $T_{N}$ cells from PBMCs may represent the most effective strategy to preserve more immature $T$ cell subsets in T cell products.

pathways that promote the precocious differentiation of $\mathrm{T}_{\mathrm{N}}$ cells. They found indeed that $\mathrm{T}_{\mathrm{MEM}}$ cells mediate the differentiation of $\mathrm{T}_{\mathrm{N}}$ cells through Fas ligand (FasL) expressed by activated $\mathrm{T}_{\text {MEM }}$ cells interacting with Fas on activated $\mathrm{T}_{\mathrm{N}}$ cells. Preventing Fas-FasL interaction by FasL-blocking antibodies or genetic ablation of Fas on $\mathrm{T}_{\mathrm{N}}$ cells restores the primitive $\mathrm{T}_{\mathrm{SCM}}$ and $\mathrm{T}_{\mathrm{CM}}$ cell populations in the $\mathrm{T}_{\mathrm{N}}$ cell progeny. In addition, provision of FasL alone is sufficient to promote $T_{N}$ cell precocious differentiation in the absence of $\mathrm{T}_{\text {MEM }}$ cells. Fas-FasL interaction induces the extrinsic apoptosis pathway via caspase 8 . Interestingly, the precocious differentiation of $\mathrm{T}_{\mathrm{N}}$ cells induced by $\mathrm{T}_{\text {MEM }}$ cells does not induce a cell death pathway, but instead depends on activation of Akt signaling and metabolic modulation. $\mathrm{T}_{\mathrm{N}}$ cells expanded with $\mathrm{T}_{\text {MEM }}$ cells show elevated activation of Akt and glycolytic activity, a phenomenon that can be recapitulated by the addition of FasL. Inhibition of Akt activation reverses the precocious differentiation, raising the possibility of pharmacologic modulation of this process.

\section{Conclusions and future directions}

It remains unknown whether there is crosstalk among the mixed populations of $\mathrm{T}_{\mathrm{EM}}$, $\mathrm{T}_{\mathrm{CM}}$, and $\mathrm{T}_{\mathrm{SCM}}$ cells present in the infused products. Moreover, since $\mathrm{CD}^{+} \mathrm{T}$ cells are also present in unfractionated PBMCs and play a role in promoting the survival of engineered $\mathrm{T}$ cells (14), determining whether this quorum-sensing phenomenon exists within the CD4 subset would greatly benefit the manufacturing of $\mathrm{T}$ cell 
products. In addition to the application in cancer immunotherapy, this uncovered crosstalk between $\mathrm{T}_{\text {MEM }}$ and $\mathrm{T}_{\mathrm{N}}$ cells may represent a physiologic immunoregulatory mechanism to favor $\mathrm{T}_{\mathrm{E}}$ cell differentiation over immune memory formation. Priming of $T_{N}$ cells in the presence of preexisting antigen-experienced $\mathrm{T}$ cells may be a signal for chronic persistent infection. Clearance of antigen by $\mathrm{T}_{\mathrm{E}}$ cells in this situation may be the priority over establishment of long-lived memory $\mathrm{T}$ cells. Moreover, since the ratio of $T_{M E M}$ to $T_{N}$ cells gradually increases with age, due to reduced output of $\mathrm{T}_{\mathrm{N}}$ cells from thymus, the discovered crosstalk between these subsets could be exploited to improve vaccination approaches in aged individuals.

Address correspondence to: Gianpietro Dotti, Department of Microbiology and Immunology, University of North Carolina; Marsico Hall, 125 Mason Farm Road, Room 5202, Chapel Hill, North Carolina
27599, USA. Phone: 919.962.8279; E-mail: gdotti@med.unc.edu.

1. Robbins PF, et al. Tumor regression in patients with metastatic synovial cell sarcoma and melanoma using genetically engineered lymphocytes reactive with NY-ESO-1. JClin Oncol. 2011;29(7):917-924.

2. Maude SL, Teachey DT, Porter DL, Grupp SA. CD19-targeted chimeric antigen receptor T-cell therapy for acute lymphoblastic leukemia. Blood. 2015;125(26):4017-4023.

3. Klebanoff CA, et al. Memory T cell-driven differentiation of naive cells impairs adoptive immunotherapy. J Clin Invest. 2016;126(1):318-334.

4. Gattinoni L, et al. Acquisition of full effector function in vitro paradoxically impairs the in vivo antitumor efficacy of adoptively transferred CD8 T cells. JClin Invest. 2005;115(6):1616-1626.

5. Gattinoni L, et al. A human memory T cell subset with stem cell-like properties. Nat Med. 2011;17(10):1290-1297.

6. Powell DJ. Transition of late-stage effector $\mathrm{T}$ cells to $\mathrm{CD} 27^{+} \mathrm{CD} 28^{+}$tumor-reactive effector memory T cells in humans after adoptive cell transfer therapy. Blood. 2005;105(1):241-250.

7. Xu Y, et al. Closely related T-memory stem cells correlate with in vivo expansion of CAR. Blood. 2014;123(24):3750-3759.

8. Brentjens RJ, et al. CD19-targeted T cells rapidly induce molecular remissions in adults with chemotherapy-refractory acute lymphoblastic leukemia. Sci Transl Med. 2013;5(177):177ra38.

9. Lee DW, et al. T cells expressing CD19 chimeric antigen receptors for acute lymphoblastic leukaemia in children and young adults: a phase 1 dose-escalation trial. Lancet. 2015;385(9967):517-528.

10. Cieri N, et al. IL-7 and IL-15 instruct the generation of human memory stem $\mathrm{T}$ cells from naive precursors. Blood. 2013;121(4):573-584.

11. Muralidharan S, et al. Activation of Wnt signaling arrests effector differentiation in human peripheral and cord blood-derived T lymphocytes. JImmunol. 2011;187(10):5221-5232.

12. Gattinoni $\mathrm{L}$, et al. Wnt signaling arrests effector $\mathrm{T}$ cell differentiation and generates $\mathrm{CD}^{+}$memory stem cells. Nat Med. 2009;15(7):808-813.

13. Wang X, Berger C, Wong CW, Forman SJ, Riddell SR, Jensen MC. Engraftment of human central memory-derived effector $\mathrm{CD}^{+} \mathrm{T}$ cells in immunodeficient mice. Blood. 2011;117(6):1888-1898.

14. Louis CU, et al. Antitumor activity and longterm fate of chimeric antigen receptor-positive T cells in patients with neuroblastoma. Blood. 2011;118(23):6050-6056. 